Military Technical College Kobry El-Kobbah, Cairo, Egypt

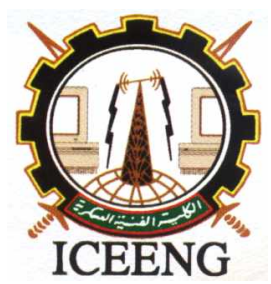

\author{
$6^{\text {th }}$ International Conference \\ on Electrical Engineering \\ ICEENG 2008
}

\title{
Laser Hazards and Ocular Exposure Analysis and Protection
}

$$
\text { By }
$$

Samy Ghoniemy*

\section{Abstract:}

In this paper, different lasers such as ruby, Argon, $\mathrm{Nd}: \mathrm{Y} \mathrm{AG}, \mathrm{He}-\mathrm{Ne}$, and $\mathrm{CO}_{2}$ are used to expose the eyes of rabbits as a simulation of the human eyes accidental laser exposure. Quantitative irradiance causing threshold damage (lesion) in as a function of wavelength under different pulse durations is studied. The retinal damage studies have shown that the threshold retinal damage decreases as increasing the exposure time. Our results also showed that the retinal sensitivity to threshold damage decreases as increasing the wavelength under different pulse duration. The basis of the physical and analytical models used to predict and analyze the laser hazards and their effect on the eye are presented. The proposed physical and analytical models include the representation of the laser and the eye characteristics are used for predicting the radiometric aspects and calculating the overall laser intensity exposing the eye. Other parameters that impact the evaluation such as atmospheric attenuation, laser wavelength, and divergence angle are considered in the proposed model. The proposed algorithm is used to predict many parameters such as hazardous distance, total incident power on the eye and the reflectance of the proposed protection filter. The simulation results of the proposed algorithm are in good agreement with the measurement results.

\section{Keywords:}

Laser effects, Laser reflection hazards, retinal laser damage, laser safety

* Egyptian Armed Forces; ghoniemy@sce.carleton.ca; Member, SPIE, OSA, IEEE 


\section{Introduction:}

With the dramatic growth in the application of lasers in everyday activities, as well as their routine utilization in scientific laboratories, industrial and military environments, many more investigators must necessarily face the matter of laser safety. Lasers have become integral components of many current optical microscopy techniques, and when combined with complex optical systems, they can constitute a significant hazard if safe procedures are not strictly followed. Recently, High Energy Laser (HEL) systems have been dramatically advances especially for military and industrial applications. Laser based new weapon systems may be risky for people because of the hazardous laser exposure during tests, training, or operational use. Accidental laser retinal injury, exposure to laser hazards, and battlefield may cause permanent vision loss. Numerous cases of accidental laser eye injuries have been reported [1]-[6]. Laser emission is similar to direct sunlight exposure in that the light arrives at the eye in parallel rays, which are very efficiently focused on the retina, the rare surface of the eye that senses light. Location of the laser lesion within the retina dependents on the laser light wavelength, beam intensity, distance from the laser, power of the laser (both average power over long intervals and peak power in CW and pulsed lasers, respectively) and energy absorbed in the tissue. Despite the increasing risk to military personnel from battlefield and accidental laser exposures, there is still no standardized evaluation and treatment regime for laser eye injuries. Additionally, there is currently no functional metric to assess recovery from laser eye injury over time that is correlated to morphologic metrics currently in use [7]. The amount of visual disruption by individuals exposed to a visible laser source at levels, which are below that, which will damage the cornea or retina will depend on laser exposure parameters and task demands. Many trials have been reported in many publications such as in [8] for studying the visual disruption experienced by individuals who have been exposed to visible laser sources under various laser exposure parameters and/or test conditions however the effect of chromic or multiple exposures is almost completely unknown. The subject of laser safety has evoked much emotional controversy over a period of years. Standards for laser safety are still in an evolutionary stage. With careful evaluation of the hazards and 
a well designed safety program, the potential danger can be reduced to an acceptable level for scientific, industrial and military use [9]. Protection in industrial or laboratory settings is normally achieved by ensuring that no unprotected persons are in the potential hazard zone and by the use of simple safety measures such as controlled entry, beam containment, and warning lights [10]. Such safety measures are sufficient to manage risk associated with laboratory and industrial use of HEL systems; however, when these systems move outdoors, for testing on open ranges, new safety issues arise and standard safety measures are impractical. Also, it is highly recommended to ensure that diffuse and specular reflections from HEL system targets, as well as direct beam paths will not harm human beings. Simple calculations become impractical when the laser source and the target are moving. In this paper we have defined the hazards to the eye in terms of number of exposure parameters such as power density for a minimum observable burn. There are some data indicating microscopic histological changes that can occur at lower levels than the burn threshold, so safety codes usually apply some safety factors. Also, in this paper we utilize a deterministic approach to laser hazard evaluation technique. This technique is used to provide an analysis of locations where hazard from direct, reflected, or scattered radiation might exist during laser operation. The suitable model is used as a simulation tool that automates HEL hazard evaluation. In this paper the physiological effects of different lasers are discussed in section II. The transmission and absorption characteristics of the eye are illustrated in section III. Section IV includes the experimental results, analysis and discussions of subjecting the eye to different lasers. The effective power calculations and eye protection are presented in Sec. V. Finally, Sec. VII includes the summary and some conclusions.

\section{Physiological Effects:}

The general anatomy of the human eye is illustrated in figure (1) [11]-[13], with emphasis on the structures that are likely to be damaged by absorption of intense radiation. The most sensitive structures of the body to the laser radiation are the retina (the photosensitive surface at the back of the eye), the cornea (the front transparent layer of the eye), and the skin. The retina can be damaged by visible $(0.38<\lambda<0.76 \mu \mathrm{m})$ 
lasers and near-infrared $(0.76<\lambda<1.4 \mu \mathrm{m})$ lasers. The light from ultraviolet $(\lambda<0.38$ $\mu \mathrm{m})$ and far infrared $(\lambda>1.4 \mu \mathrm{m})$ lasers does not reach the retina, but can damage the cornea while the skin can be affected by lasers of any wavelength [14].

Only light in the range from 0.38 to $1.4 \mu \mathrm{m}$ can penetrate through the interior structures of the eye and reach retina. Interaction of laser beams and the retina have been studied by a number of investigators [11], [12], [15]-[18]. It has been shown that at the power levels used for interactions of minimal damage and for clinical applications, very little of energy is absorbed by the photopigments of the eye. The pigment epithelium and choroids absorb the majority of the energy that reaches the funds. The resulting interaction causes thermal damage to the tissues in the funds of the eye. Because of its high density of melanin granules, the pigment epithelium is the place where the highest absorption per unit volume takes place. The highest temperature rise in the back of the eye will thus take place near or inside the pigment epithelium layer. Because of the geometry of the absorbing layers, the thermal conduction can take place away from the interaction region. The size of the interaction area plays a very important role as far as temperature rise and decay of the exposed site are concerned [9].

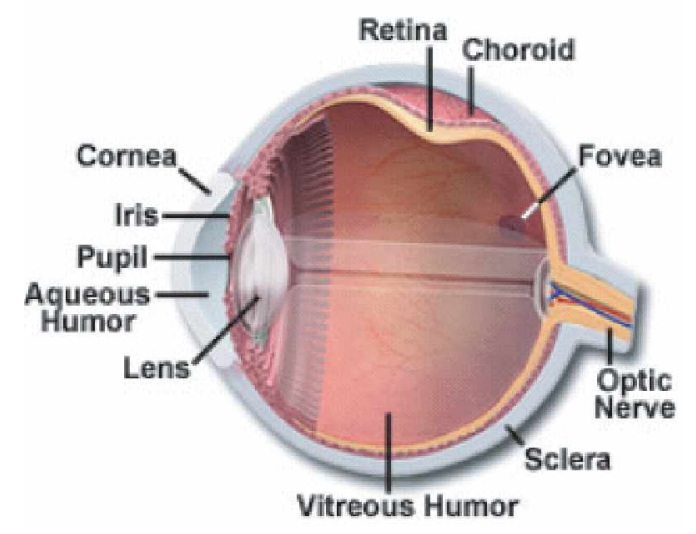

Figure (1): Anatomy of the human eye.

\section{Eye Characteristic And Hazards:}

The physical response of the human eye differs for light of different wavelengths, and this has a bearing on the potential damage that may occur for several reasons that will be discussed below. It is notable that a common warning for most categories of laser is 
to avoid viewing the beam with any magnifying optical device. A primary danger to the human eye posed by lasers results from the fact that the eye itself is a highly precise and efficient focusing optical device for light within a certain wavelength range. Utilizing lasers in conjunction with microscope optics increases the potential of the eye damage. It is common for optics laboratories to contain many lasers, both as integrated components of systems such as fluorescence microscopes, and as light sources on open optical benches. The major dangers presented by these open lasers are potential damage to the eyes from stray horizontal beams at table height, beams reflected out of the plane of the table, and reflections from optical components and external reflective surfaces, such as belt buckles, watches, jewelry, and any reflective surfaces in the room. Even a split second exposure to a small reflected portion of a laser beam may be sufficient to cause permanent injury and loss of vision. The potential for laser emission causing injury to the different structures of the eye depends upon which structure absorbs the beam energy. The absorption characteristics of the different eye tissues, and the wavelength and intensity of the laser light determine whether damage occurs to the cornea, lens, or retina. The wavelengths that penetrate to the retina at the rear eye surface are determined by the overall transmission characteristics of the eye. The transmission characteristic of the ocular media in terms of incident radiation is shown in Figure (2) [17], [18]. Figure (2) essentially defines the hazards to the retina as a function of wavelength. These curves are based on Vitro measurement of the human eyes. Figure (2) illustrates that the ocular media is a good transmitter for the visible and near infrared radiations. Therefore, radiation whose wavelength fall in this range will reach the retina with very little loss to the ocular media. Wavelengths in the infrared region, however, are highly absorbed because of the water contents in the media. This figure also shows the absorption characteristics of the melanin granules (retina) responsible for the major part of the absorption in the pigment epithelium and choroids (or it shows the product of transmission through the ocular media and absorption in the various layers of the retina). If the light is intense enough, it can damage the structures in which it is absorbed, usually the cornea. Also, it is observed that the most effective wavelengths that can heat the pigment epithelium are those fall in the blue-yellow region of the visible spectrum. The optical gain of the relaxed human eye for a highly collimated 
beam, which is the ratio of the area of the eye's pupil to the retinal (focused) image area, is on the order of 100,000 . This corresponds to five orders of magnitude irradiance increase from the corneal surface to the retina. Since a collimated laser beam entering the eye would be focused to a small area on the retina, the power density in the focal spot would be much higher than that of the incident beam at the entrance of the eye. Since the diameter of the beam on the retina could be approximately $20 \mu \mathrm{m}$, a very large increase in power density is possible [19] since the power density is described by $\left(d_{p} / d_{r}\right)^{2}$, where $\mathrm{d}_{\mathrm{p}}$ is the diameter of the pupil of the eye and $\mathrm{dr}$ is the focal diameter of the spot on the retina. A representative case might be $d_{p} \approx 0.5 \mathrm{~cm}$ and $d_{r} \approx 20 \times 10^{-4} \mathrm{~cm}$ this would yield a peak power density on the retina about $6 \times 10^{4}$ times larger than the power density entering the eye. In this case if a laser beam entering the eye directly (intra-beam viewing), a 1milliwatt beam produces a retinal irradiance value on the order of 60 watts per square centimeter. In comparison, direct viewing of the sun produces irradiance at the retina of approximately $10 \mathrm{~W} / \mathrm{cm}^{2}$. Therefore, the retina could be damaged at certain levels where other structures of the body would not be endangered. The seemingly low rated power of lasers can be very misleading with respect to the damage possible when the energy is concentrated to this extent.

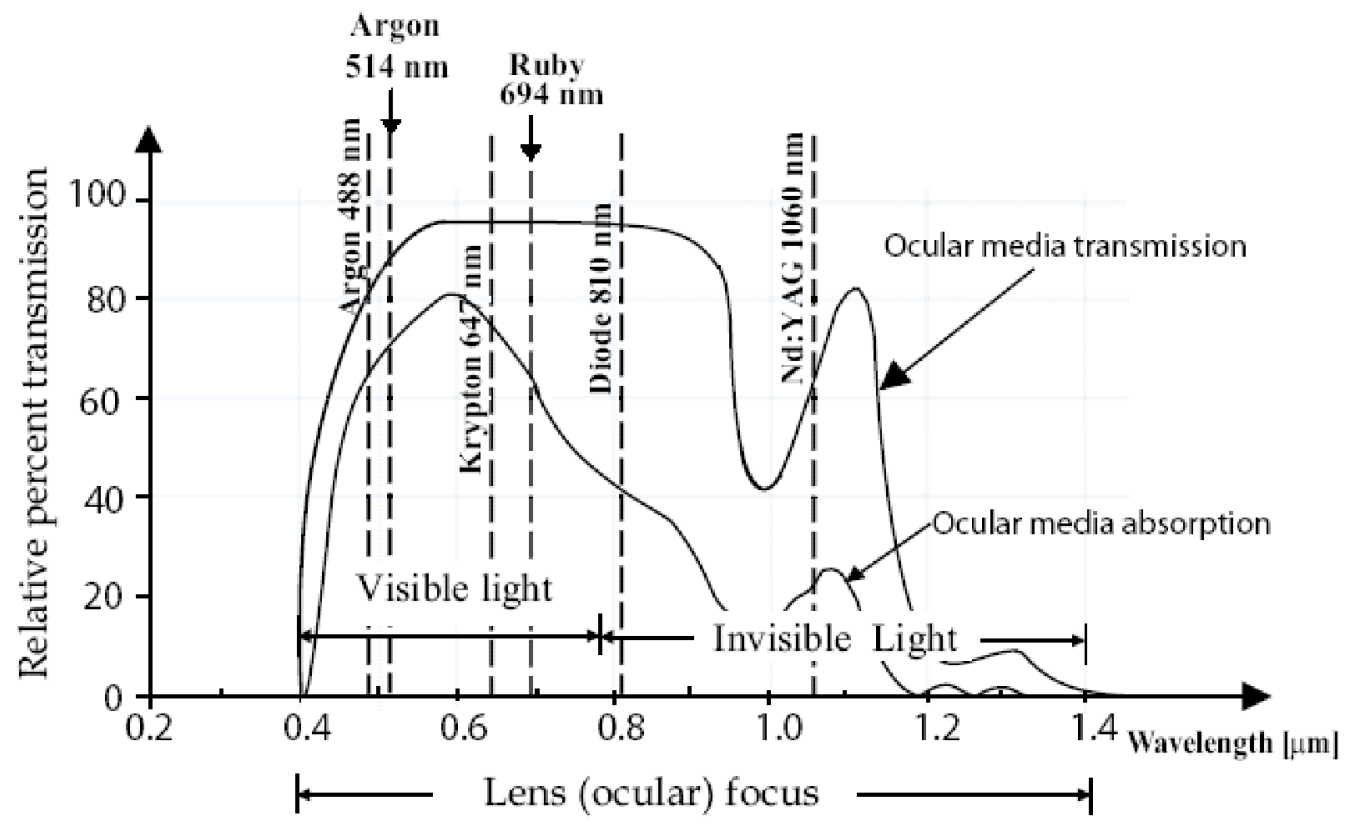

Figure (2): Spectral characteristics of the human eye. The curve gives the percentage transmission through the anterior structure of the eye (Ocular). 


\section{Experimental Methods And Results:}

In these experiments ruby, argon, $\mathrm{Nd}: \mathrm{YAG}, \mathrm{He}-\mathrm{Ne}$, and $\mathrm{CO}_{2}$ lasers are used with different pulse rates and power densities. In some experiments a continuous Nd:YAG laser source is used. In this study 40 rabbits were used.

\section{A. Experiment 1}

In this experiment retinal lesions were produced by a ruby laser source emitting at the red end of the visible spectrum simulating human accidental laser shock to the central funds. Figure (3) shows the experimental setup for specifying the threshold power causing retinal burns. In these experiments, the left eye of 20 rabbits is exposed to direct exposures. The immediate lesion size/diameter that was involved was variable, typically ranging from 10 to $800 \mu \mathrm{m}$ of the disk diameter. Laser pulse energy is measured using a Molectron Joule meter. A translation stage is used to move the rabbit eye along the focused beam. An automated beam profiler is used to measure and characterize the beam diameter. Figure (4) shows the effect of the laser power on the retina under different image sizes and spot radius to specify the minimal power that causes retinal lesions or burns under different exposure durations.

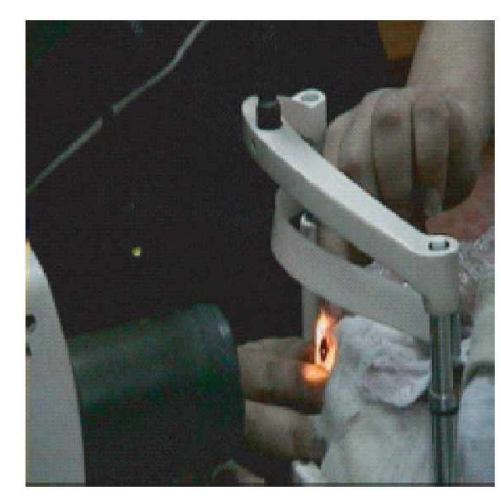

Figure (3): TRC50A instrument to observe the rabbit eye lesions after exposure 


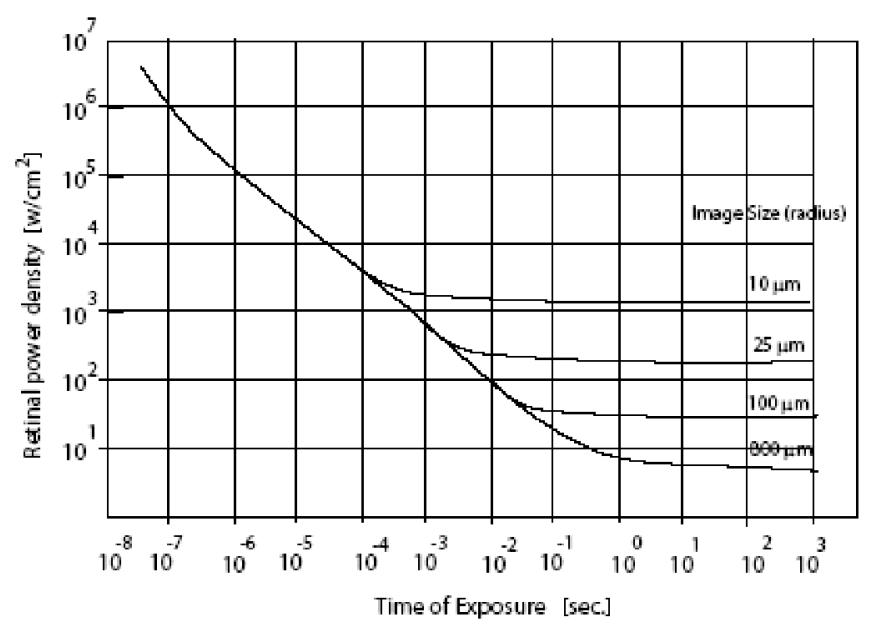

Figure (4): Threshold power density needed to cause minimum ophthalmoscopically observed lesion on the retina of rabbit eyes as a function of exposure time for different retinal image sizes.

Figure (4) determines the damage threshold power density in $\mathrm{W} / \mathrm{cm}^{2}$. It is observed that the threshold power for an observable lesion is a strong function of both pulse duration and retinal image size. In our research the threshold is defined as the value at which the observers could barely detect small burns when observing the retina through an ophthalmoscope. It is also noticed that higher energy values than the threshold causes serious damage, including production of large burns and craters in the retina, ejection of retinal material into the vitreous medium of the eye, and hemorrhage within the eyeball.

\section{B. Experiment 2}

Another set of experiments are performed using rabbits exposed to an Argon laser of wavelength 488. Figure (5) shows the experimental results of this experiment when the image sizes are 10, 50, and $100 \mu \mathrm{m}$. As illustrated in Figure (5), the threshold power is a decreasing function over time for all image sizes and laser wavelengths. These results do not fully match the theoretically predicted results using simple thermal models. This discrepancy is due to the fact that the damage is caused by protein denaturation or inactivation of enzymes. Because of the rate relationship associated with these processes, the length of time of keeping the tissues at certain temperature (exposure time) is an important factor. Hence, there is no unique critical temperature in case of short interaction. For a longer interaction; the same effect can be generated by using a 
slightly lower temperature which means that a lower power laser can cause the same effect when tissues are exposed to that laser for longer time. To assess the effects of laser induced acute and long term damage on the human color vision, it is necessary to select color vision metrics that are capable of detecting the fundamental characteristics of color vision and its unique role in human vision. One of the approaches to attack this problem of diagnostics is to select color vision metrics that rate highly sensitive to three cone photoreceptor systems. Nonhuman primates (NHP) demonstrate that selective cone damage effects can be detected in measures of spectral sensitivity and spectral acuity [20]. The more recent metrics of human color vision assessment emphasizing identification of different systems using chromatic acuity chart systems have demonstrated a similar capability under human clinical conditions [21]. On the other hand, the use of these metrics does not evaluate the higher level of cone system integration required for fine chromatic discrimination across the visible spectrum [13].

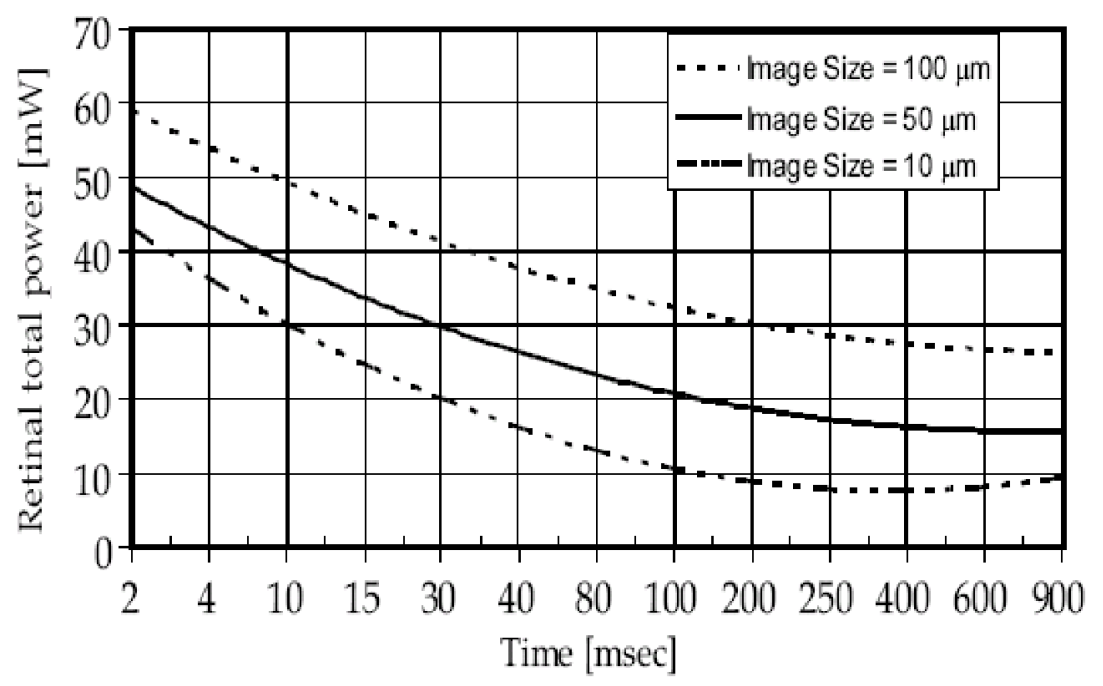

Figure (5): Threshold retinal damage in rabbits by Argon laser photocoagulation

\section{Experiment 3}

Five rabbits were used and retinal lesion were produced by Nd:YAG laser $(1.064 \mu \mathrm{m})$. Exposures under different powers are used and the averaged results are demonstrated in figure (6). The trend of using the Nd:YAG laser is similar to that for an Argon laser except that the same effect can be observed but higher power is required (typically, the needed power while using Nd:YAG is five times higher than if the Argon laser is used). 
This can be clarified by observing the eye characteristic shown in figure (2), from which it is noticed that the radiation of wavelength $1.06 \mu \mathrm{m}$ is one of the highly absorbed wavelength by the ocular media.

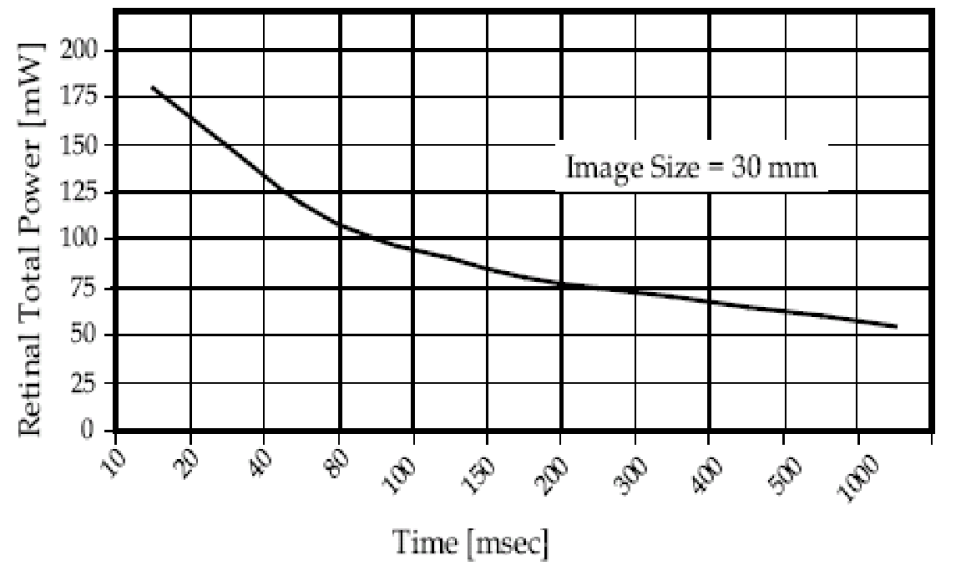

Figure (6): Threshold retinal damage in rabbits by Nd:YAG laser photocoagulation

\section{Experiments $4 \& 5$}

The same experiments are done using He-Ne laser source and the results are illustrated in figure (7). Only lesions are observed when the power is approximately greater or equal to $7 \mathrm{~mW}$ for long exposure time.

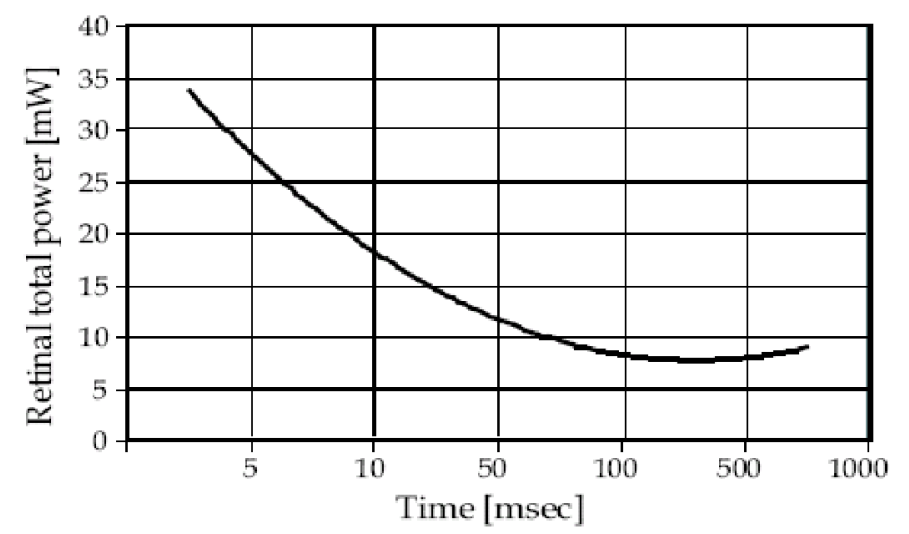

Figure (7): Threshold values of He-Ne laser power needed to cause minimum retinal lesions in rabbits as a function of exposure duration

\section{E. Experiment 6}

Another set of experiments are done by subjecting set of rabbits to a $\mathrm{CO}_{2}$ laser operating at $10.6 \mu \mathrm{m}$. Radiations of this wavelength don't penetrate to the retina. It is absorbed at 
the cornea and can cause corneal burn. For minimal burns near threshold, the effect is to cause opacity of cornea, which heals within a few days. Figure (8) shows the experimental data on the threshold $\mathrm{CO}_{2}$ laser power density required to cause corneal opacities. Taking together figures 5-to-8, we conclude that for equal beam powers a visible laser is likely to be more dangerous than infrared laser because of the focusing action of the eye. This can be clarified by knowing that the power at retina is higher than that at the cornea.

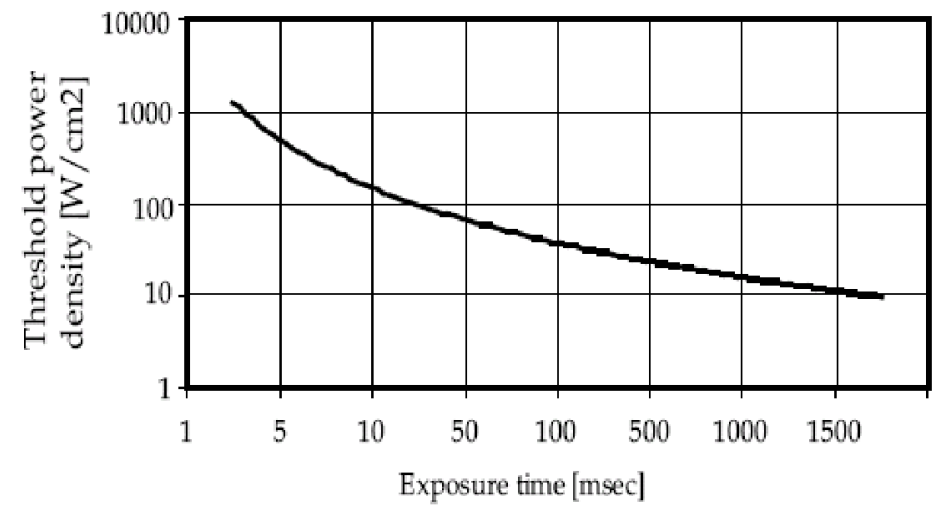

Figure (8): Threshold values of CO2 laser power needed to cause minimum damage to the cornea of rabbits as a function of exposure duration

\section{F. Experiment 7}

The reciprocal of the threshold retinal irradiance required to produce a minimum retinal burn in rabbits under several exposure times are shown in figure (9) to investigate the retinal sensitivity to the threshold damage as a function of wavelength.

Figure (9) shows that the threshold irradiance using blue lasers is much lower than that of using red lasers. The difference can't be treated as a pure thermal since photochemical action may influence the damage when using blue lasers. This leads to conclude that blue lasers are more dangerous to the retina than red lasers at the same power level. At last, the extrapolation of data from rabbit or other animals such as rhesus monkey eyes to human eyes is not identical and therefore, the effects of laser light on the ability of the human eyes to see have not been completely established, however it is a suitable indication for the determination of the minimum power causes damages to the human eye. 


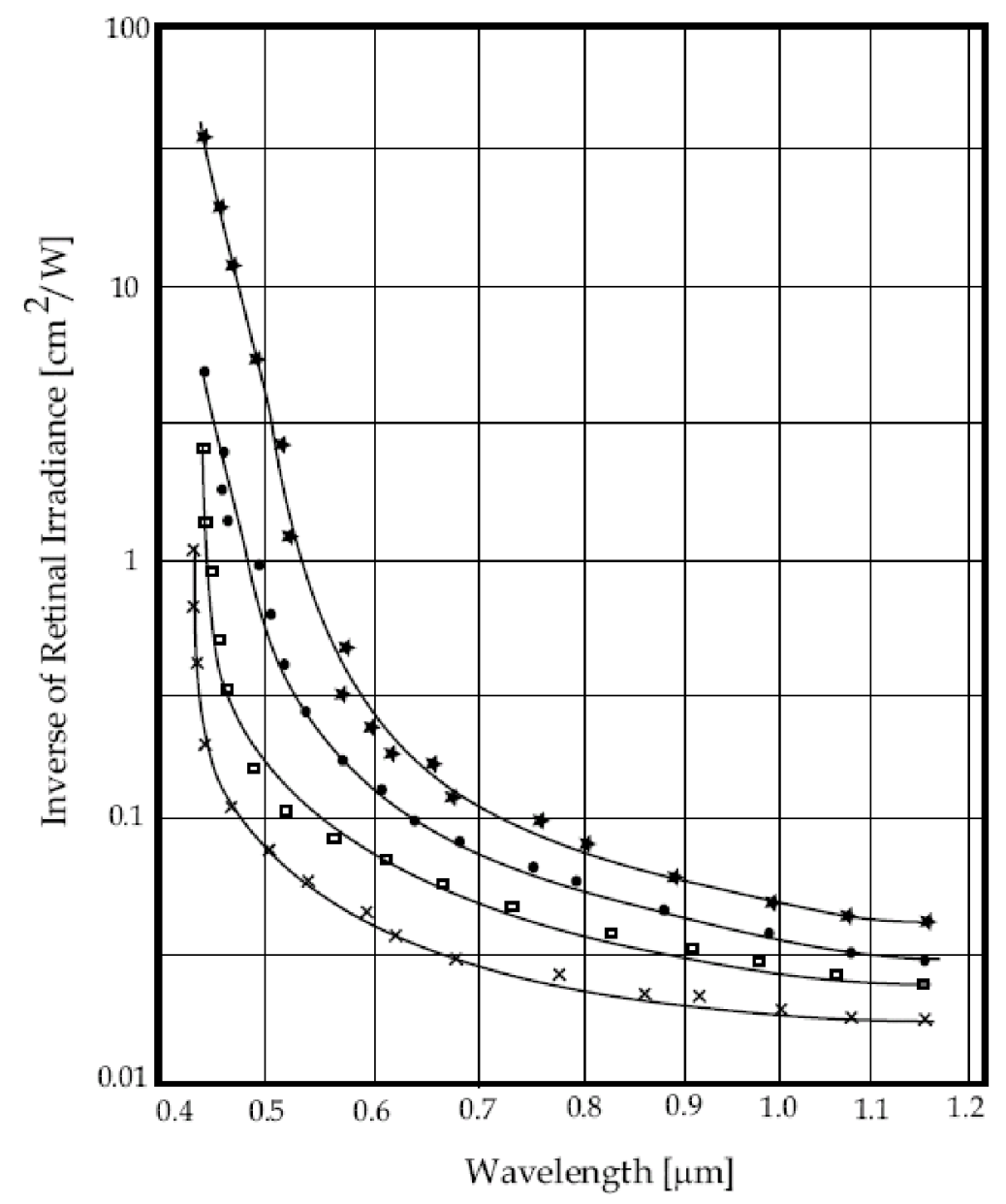

Figure (9): Reciprocal of irradiance need to cause threshold damage (lesion) in rhesus monkeys as a function of wavelength under different pulse durations $(\times 1$ sec., $\quad 12$ sec., $\bullet 50$ sec., and $* 750$ sec.)

Taking all together, experimental results in figures 4-to-9 show that the potential damage to the eye can be categorized with respect to the laser wavelength and the eye structures affected by radiation in the visible and near-infrared spectral region. Thermal burn or photochemical alteration is possible depending upon the energy absorbed. The biological effects on the eye tissues manifested within various wavelength bands are summarized and listed in Table I. 
Table (I): Rule base for the position controller

\begin{tabular}{|l|l|c|}
\hline \multicolumn{1}{|c|}{ Skin effects } & \multicolumn{1}{|c|}{ Eye effects } & Spectral band \\
\hline $\begin{array}{l}\text { • skin burn } \\
\text { - photosensitive } \\
\text { reactions }\end{array}$ & $\begin{array}{l}\bullet \text { Photochemical, } \\
\bullet \text { Thermal retinal injury color } \\
\bullet \text { Night vision degradation }\end{array}$ & Visible $(400780 \mathrm{~nm})$ \\
\hline$\bullet$ skin burn & $\bullet$ Retinal burns cataract & Near Infrared (780 1400 nm) \\
\hline$\bullet$ skin burn & $\begin{array}{l}\bullet \text { Corneal burn } \\
\bullet \text { Aqueous flare IR cataract }\end{array}$ & Middle Infrared (14003000 nm) \\
\hline$\bullet$ skin burn & $\bullet$ Corneal burn & Far Infrared (3000 106 nm) \\
\hline
\end{tabular}

\section{Effective Power Computation Algorithm And Eye Protection:}

Laser safety measures and utilities are varied from physical barriers to engineering controls. For eye protection, the barriers are optical materials that minimize as much as possible the amount of energy at that wavelength to reach the eye. The most common eye protection method is to use optical filters [22]. A measure of the ability of an optical component to withstand high laser irradiation is its laser induced damage threshold (LIDT). In our research optical interference filters are used to protect human eyes by preventing laser energy larger than the damage threshold. Using the threshold damage power presented in Sec. IV and the characteristics of a laser source, filter reflectance be predicted according to the proposed algorithm presented as follow:

Any laser source is characterized by the following parameters:

Average/Peak power, $\mathrm{P}_{\text {source}}$, wavelength (or range of wavelengths), $\lambda_{\text {source, }}$ divergence angle of the source, $\theta_{\text {source}}$, power spectrum with respect to wavelength.

Using these parameters, the power density, irradiance, before the protective filter $H_{b}$ is calculated using,

$$
\mathrm{H}_{\mathrm{b}}=\frac{\mathrm{P}_{\text {source }}}{A}
$$

$\mathrm{A}$ in which, $\mathrm{A}=2 \pi \mathrm{r}^{2}$, is the area of the image size (laser spot), and $\mathrm{r}$ is its radius. The 
spot area can be approximated by $\mathrm{A}=\pi\left(\mathrm{R} \times \theta_{\text {source }}\right)^{2}$, in which $\mathrm{r} \approx \mathrm{R} \times \theta_{\text {source, }}$, where $\mathrm{R}$ is the distance between the laser source and the eye. This leads to express $\mathrm{H}_{\mathrm{b}}$ as,

$$
\mathrm{H}_{\mathrm{b}}=\frac{\mathrm{P}_{\text {source }}}{\pi\left(\mathrm{R}^{2} \times \theta_{\text {source }}^{2}\right)}
$$

The power density after the protective filter is given by,

$$
H_{a}(\lambda)=H_{b}(\lambda) T(\lambda)
$$

in which $T(\lambda)$ is the spectral transmittance of the optical filter. All together lead to calculate the incident power on the human eye and given by,

$$
P_{\text {eye }}(\lambda)=H_{a}(\lambda)(\tau(\lambda) \alpha(\lambda)) A_{\text {eye }}
$$

in which $\tau(\lambda)$ is the ocular media transmission and $\alpha(\lambda)$ is the pigment epithelium and choroids absorption for each wavelength as shown in figure (2), and $A_{\text {eye }}$ is the area of the pupil of the eye. By knowing the exposure time period, $\Delta \mathrm{t}$, and specifying the threshold power, $P_{t h}$, of the eye at a specified wavelength using the presented results in section IV, the effective total power incident on the eye can be predicted. If the incident power on the eye is greater than or equal to the threshold power, this means that the filter reflectance is not sufficient to protect the eye at that wavelength and another highly reflective filter should be used. Otherwise, the proposed filter is suitable.

\section{Algorithm Implementation And Results:}

The proposed algorithm is implemented using C\#.net language. The inputs to the program are the source parameters, the distance between the source and the eye, the ocular media transmission, the pigment epithelium and choroids absorption, and the atmospheric transmission factor. To examine the ability of the proposed algorithm to predict the effective power on the human eye and to propose the suitable filter's reflectance under different distances between the source and the eye, an Argon laser source emitting at wavelength $\lambda_{\text {source }}=514 \mathrm{~nm}$, its output power is $P_{\text {source }}=10 \mathrm{~W}$ with divergence angle $\theta_{\text {source }}=1 \mathrm{mrad}$ is used. Assuming that the time of exposure $\Delta t=100 \mathrm{msec}$, while we assumed that the image size is $a=15 \mu \mathrm{m}$, and the size of the pupil of the eye is $d_{p}=4.37 \mathrm{~mm}$ (constant). Therefore, the area of the eye approximately 
equal $15 \mu \mathrm{m}^{2}$.

From figure (2), the ocular media transmission $\tau(\lambda)$ approximately equal $90 \%$, and the pigment epithelium and choroids absorption $\alpha(\lambda)$ approximately equal $80 \%$. Using these parameters the program predicts the effective power. Table II shows the predicted effective power incident on the eye at different distances as a function of the filter transmittance. To demonstrate these results, the calculated total power incident on the eye is compared with the threshold power. From figure (5), at $100 \mathrm{msec}$ exposure time, the threshold power approximately equal $12 \mathrm{~mW}$. Comparing the threshold power with $P_{\text {eye }}$ when the filter reflectance at this wavelength is greater than or equal $98 \%$. The result of this comparison shows that the hazardous distance is $\leq 2 \mathrm{Km}$.

Table (II): the predicted effective power incident on the eye at different distances as a function of the filter transmittance.

\begin{tabular}{|l|c|c|c|c|}
\hline \multicolumn{1}{|c|}{$\mathrm{P}_{\text {eye }}$} & $\alpha(\lambda)$ & $\tau(\lambda)$ & $\mathrm{H}_{\mathrm{b}}$ & $\mathrm{R}$ \\
\hline $3.44 \times 10^{-2} \times \mathrm{T}$ & $80 \%$ & $90 \%$ & $3.18 \times 10^{4}$ & $10 \mathrm{~km}$ \\
\hline $5.374 \times 10^{-2} \times \mathrm{T}$ & $80 \%$ & $90 \%$ & $4.98 \times 10^{4}$ & $8 \mathrm{~km}$ \\
\hline $9.55 \times 10^{-2} \times \mathrm{T}$ & $80 \%$ & $90 \%$ & $8.85 \times 10^{4}$ & $6 \mathrm{~km}$ \\
\hline $2.15 \times 10^{-1} \times \mathrm{T}$ & $80 \%$ & $90 \%$ & $1.99 \times 10^{5}$ & $4 \mathrm{~km}$ \\
\hline $8.6 \times 10^{-1} \times \mathrm{T}$ & $80 \%$ & $90 \%$ & $7.96 \times 10^{5}$ & $2 \mathrm{~km}$ \\
\hline $3.44 \times 100 \times \mathrm{T}$ & $80 \%$ & $90 \%$ & $3.18 \times 10^{6}$ & $1 \mathrm{~km}$ \\
\hline
\end{tabular}

\section{Summery and Conclusions:}

An exposure setup is used to measure the threshold for laser induced retinal damage in the rabbit eyes using different laser sources under different times of exposure. Animals are sedated and anesthetized for exposure. Full pupil dilation was pharmaceutically induced in the eye to be exposed. For each experiment, the exposure sites are examined and the presence of a minimum visible lesion is noted for each site. The results presented in this paper are based on animal experiments using rabbits. Different lasers such as ruby, Argon, $\mathrm{Nd}: \mathrm{YAG}, \mathrm{He}-\mathrm{Ne}$, and $\mathrm{CO}_{2}$ with different powers and under different exposure times are used in these experiments to study and analyze the effect of laser hazards on the human eye. Analyzing these results, the eye optics plays a critical role in localizing the laser induced thermal retinal damage to the retinal pigment 
epithelium because it lies at its focus. The action of exposing the eye to any laser source is modeled and a mathematical algorithm for predicting the total incident power on the eye is discussed. The model has been solved using C\#.net computer language and a case study using Argon laser is presented. Optical interference filters are introduced as a safe protection tool when eyes are subjected to laser energy larger than the threshold energy. It is also concluded that as the exposure time increases sever effects are occurred. Using the proposed algorithm, the maximum exposure time for a given range and power of the source can be predicted. Also, at a given distance and type of hazardous source, the proposed protection filter's reflectance can be predicted. At the end, all the results presented in this paper are based on animal experiments; therefore, these threshold values can be relevant for the establishment of laser safety codes.

\section{References:}

[1] A. B. Thach, P. F. Lopez, L. C. SandyMcCoy, B. M. Golub, and D. A. Frambach, "Accidental Nd:YAG laser injuries to the macula," Am J. Ophthalmol, vol. 119, no. 6, pp. 767-773, 1995.

[2] D. R. Hirsch, D. G. Booth, S. Schocket, and D. H. Sliney, "Recovery from pulseddye laser retinal injury," Arch Ophthalmol, vol. 110, no. 12, pp. 16881689, 1992.

[3] T. A. Ciulla and T. M. Topping, "Surgical treatment of a macular hole secondary to accidental laser burn," Arch Ophthalmol, vol. 115, no. 7, pp. 929-930, 1997.

[4] S. Ray, T. Topping, and L. H. Young, "Spontaneous peeling of epiretinal membrane associated with Nd:YAG laser injury," Arch Ophthalmol, vol. 119, no. 1, pp. 137-139, 2001.

[5] J. K. Lutrull and J. Hallisey, "Laser PointerInduced macular injury," Am J. Ophthalmol, vol. 127, no. 1, pp. 95-96, 1999.

[6] J. Roider and et. al., "Macular injury by a military range finder," Retina, vol. 19, no. 6 , pp. 533-544, 1999.

[7] D. C. Hood, W. Seiple, K. Holopigian, and V. Greenstein, "A comparison of the components of the multifocal and fullfield ERGs." Vis Neurosci, vol. 14, no. 3, pp. 533-544, 1997.

[8] D. A. Stamper, D. Lund, J. W. Molchany, and B. E. Stuck, "Human pupil and eyelid response to intense laser light: implications for protection," Perceptual and Motor skills, vol. 95, pp. 775-782, 2002.

[9] S. Ghoniemy, "Design and Production of AntiLaser ThinFilm Multilayer Interference Optical Filters," Master's thesis, MTC, October 1996. 
[10] FDA web page, "Notice to industry," http://WWW.fda.gov/cdrh/teprsc.html.

[11] J. A. Wolfe, "Laser Retinal Injury," Military Medicine, vol. 150, pp. 177-185, 1985.

[12] N. Ogata, J. TombranTink, N. Jo, D. Mrazek, and M. Matsumura, "Upregulation of pigment epithelium derived factor after laser photocoagulation," Am J. Ophthalmol, vol. 132, pp. 427-429, 2001.

[13] C. W. Oyster, The Human Eye. Sunderland Mass.: Sinauer Associates, 1999.

[14] J. F. Ready, Industrial Applications of Lasers. Academic Press Inc., NY, San Francisco, London, 1987.

[15] J. A. Zuclick and et. al., "Laser Induced RetinalDamage Threshold as a Function of Retinal Image Size," in Ophthalmic Technologies 1X, SPIE, Ed., vol. 3591, January 1999, pp. 335-343.

[16] D. Smith, "Protecting Eyes and Instruments from Laser Radiation," United States Patent 5831769, Nov. 31998.

[17] J. Marshall, "Structural Aspects of LaserInduced Damage and their Functional Implications," Health Physics, vol. 56, pp. 617-624, 1989.

[18] D. Land and P. Edsall, "Action Spectrum for Retinal Thermal Injury in Lasers and Non Coherent Light Ocular Effects," SPIE, vol. 3591, pp. 324-334, 1999.

August 28, 2007 DRAFT

[19] F. A. L'Esperance, Ophthalmic Lasers, 3rd ed. C. V. Mosby Comp., 1989.

[20] H. Zwick, "Visual function changes associated with lowlevel light effectsA review," Health Physics, vol. 56, pp. 657 - 663, 1989.

[21] J. Rabin, "Cone specific measures of human color vision," Investigation Ophthalmology and Visual Science, vol. 37, no. 13, pp. 2771-2274, 1996.

[22] S. Ghoniemy, "Design and Production of AntiLaser ThinFilm Multilayer Interference Optical Filters,"” M.Sc., MTC, Egypt, Oct. 1996. 